\title{
Regulation of the tumor suppressor PTEN by SUMO
}

\author{
J González-Santamaría ${ }^{1}$, M Campagna ${ }^{1}$, A Ortega-Molina ${ }^{2}$ L Marcos-Villar ${ }^{1}$, CF de la Cruz-Herrera ${ }^{1}$, D González ${ }^{1}$, P Gallego ${ }^{1}$, \\ F Lopitz-Otsoa ${ }^{3}$, M Esteban ${ }^{1}$, MS Rodríguez ${ }^{3,4}$, M Serrano ${ }^{2}$ and C Rivas ${ }^{*, 1}$
}

The crucial function of the PTEN tumor suppressor in multiple cellular processes suggests that its activity must be tightly controlled. Both, membrane association and a variety of post-translational modifications, such as acetylation, phosphorylation, and mono- and polyubiquitination, have been reported to regulate PTEN activity. Here, we demonstrated that PTEN is also posttranslationally modified by the small ubiquitin-like proteins, small ubiquitin-related modifier 1 (SUMO1) and SUMO2. We identified lysine residue 266 and the major monoubiquitination site 289, both located within the C2 domain required for PTEN membrane association, as SUMO acceptors in PTEN. We demonstrated the existence of a crosstalk between PTEN SUMOylation and ubiquitination, with PTEN-SUMO1 showing a reduced capacity to form covalent interactions with monoubiquitin and accumulation of PTEN-SUMO2 conjugates after inhibition of the proteasome. Moreover, we found that virus infection induces PTEN SUMOylation and favors PTEN localization at the cell membrane. Finally, we demonstrated that SUMOylation contributes to the control of virus infection by PTEN.

Cell Death and Disease (2012) 3, e393; doi:10.1038/cddis.2012.135; published online 27 September 2012

Subject Category: Cancer

The PTEN (phosphatase and tensin homolog deleted for chromosome 10) tumor suppressor gene, located at human chromosome $10 \mathrm{q} 23$, is frequently mutated in a number of tumor types, including glioblastoma, melanoma, and carcinomas of the prostate, breast, and endometrium. ${ }^{1-3}$ PTEN is a phosphatase antagonizing the actions of phosphoinositide 3-kinase (PI3K) by dephosphorylating the lipid second messenger phosphatidylinositol 3,4,5-triphosphate, at the plasma membrane, ${ }^{4-7}$ thus opposing the activation of the AKT kinase and its downstream cellular survival and growth responses. ${ }^{8-11}$ Although its membrane association is essential for its lipid phosphatase activity, there are only a few specific situations where PTEN shows membrane localization. PTEN also possesses numerous biological functions independent of its lipid phosphatase activity. These include regulation of cell migration, cell cycle transition, chromosomal integrity and virus replication. ${ }^{12-18}$ The crucial function of PTEN in multiple cellular processes suggests that the enzyme needs to be tightly regulated. PTEN is indeed controlled by both, membrane association and multiple post-translational modifications, such as acetylation, phosphorylation, and mono- and polyubiquitination. ${ }^{19}$

Attachment of small ubiquitin-related modifier (SUMO) to target proteins is an important post-translational regulatory mechanism. Mammalian cells express SUMO1 and the highly-related proteins SUMO2 and SUMO3. These proteins are structurally related to ubiquitin and are covalently attached to target proteins by a SUMO-conjugation system consisting of an E1 activating enzyme (SAE1/SAE2), an E2 ligase (UBC9, also known as UBE2I), and various E3 ligases with differing target-protein specificities. ${ }^{20,21}$ SUMO conjugation controls diverse cellular functions, ${ }^{20-22}$ sometimes through counteracting or contributing to ubiquitin conjugation. ${ }^{23,24}$ Thus, SUMO1 modification serves to protect Smad4 or the $\mathrm{NFkB}$ (nuclear factor $\mathrm{kB}$ ) regulator IkB $\alpha$ (inhibitory $\mathrm{kB} \alpha$ ) from ubiquitin-dependent degradation. ${ }^{25,26}$ In contrast, SUMOylation of NEMO (NF-kB essential modulator) that results in response to genotoxic stress allows its accumulation in the nucleus, thus facilitating its phosphorylation by the DNAdamage-induced kinase ATM (ataxia telangiectasia mutated), and allowing its subsequent ubiquitination at the same lysines originally targeted for SUMOylation to ultimately activate IKK (IkB kinase) and NFkB. ${ }^{27}$ SUMO and ubiquitin can also act sequentially in the regulation of the promyelocytic leukemia protein (PML). Formation of SUMO2/3 chains recruits the SUMO-binding ubiquitin ligase RNF4 (ring finger protein 4), which mediates the ubiquitination of the SUMO chains, targeting the PML protein for degradation. ${ }^{28-30}$

Here, we demonstrated that PTEN is regulated by SUMOylation. We identified the major SUMO attachment lysine residues in PTEN and we evaluated the interplay between this SUMOylation and ubiquitin conjugation. We also discovered that virus infection induces PTEN SUMOylation and its membrane localization, and finally we demonstrated

\footnotetext{
${ }^{1}$ Centro Nacional de Biotecnología, CSIC, Campus Universidad Autónoma de Madrid, 28049 Madrid, Spain; ${ }^{2}$ Centro Nacional de Investigaciones Oncológicas, Melchor Fernández Almagro, 28029 Madrid, Spain; ${ }^{3}$ Proteomics Unit, CIC bioGUNE, CIBERehd, Bizkaia Technology Park. Building $801 \mathrm{~A}, 48160$ Derio, Spain and ${ }^{4}$ Ubiquitylation and Cancer Molecular Biology laboratory, Inbiomed, San Sebastian-Donostia, 20009 Gipuzkoa, Spain

*Corresponding author: C Rivas, Centro Nacional de Biotecnología, CSIC, Campus Universidad Autónoma de Madrid, Darwin 3, 28049 Madrid, Spain. Tel: +34 915855303; Fax: +34 915854506; E-mail: crivas@ cnb.csic.es

Keywords: PTEN; SUMO; VSV

Abbreviations: PTEN, phosphatase and tensin homolog deleted for chromosome 10; SUMO1, small ubiquitin-related modifier 1; SUMO2, small ubiquitin-related modifier 2; PI3K, phosphoinositide 3-kinase; NFkB, nuclear factor kB; IkB $\alpha$, inhibitory subunit of NFkB; NEMO, NF-kB essential modulator; ATM, ataxia telangiectasia mutated; IKK, IkB kinase; PML, promyelocytic leukemia protein; RNF4, ring finger protein 4; Ub, ubiquitin; Ub-KO, ubiquitin K all R mutant; VSV, Vesicular stomatitis virus; MEFs, mouse embryo fibroblasts

Received 02.7.12; revised 24.8.12; accepted 27.8.12; Edited by A Stephanou
} 
that SUMOylation contributes to the control of virus infection by PTEN.

\section{Results}

PTEN is modified by SUMO. In silico analysis of the PTEN sequence revealed different lysine residues susceptible to work as SUMO acceptors. In addition, PTEN was shown previously to associate with the SUMO-conjugating enzyme Ubc9. ${ }^{31}$ For this reason, we decided to evaluate the putative conjugation of PTEN to SUMO. In vitro SUMOylation assays were done using recombinant PTEN protein, or in vitro translated $\left[{ }^{35}\right.$ S $]$ methionine-labeled PTEN protein, as a substrate. We detected PTEN protein as a single band of the expected 55-kDa predicted molecular weight. When the reaction was incubated with SUMO1, we observed higher molecular weight bands of around $70-75 \mathrm{kDa}$, and a faint band of around $100 \mathrm{kDa}$ (Figure 1a). In addition, when the reaction was incubated with $\mathrm{SUMO} 2$, we visualized a thinner band of $70-75 \mathrm{kDa}$ and additional higher molecular weight bands (Figure 1a). These results indicate that PTEN is modified by SUMO1 and SUMO2 in vitro. To further prove that the bands correspond to PTEN-SUMO conjugates, we incubated PTEN-SUMO1 protein with the recombinant
deSUMOylase enzyme SENP1. The high molecular weight bands detected in the lane corresponding to PTEN-SUMO1 disappeared after incubation of the reaction with SENP1 (Figure 1b). All together, these results demonstrate that PTEN is SUMOylated in vitro by SUMO1 and SUMO2. In addition, the presence of several bands corresponding to SUMO1-PTEN in the in vitro assay indicates that SUMOylation occurs at more than one site.

Then, to determine whether PTEN also conjugates to SUMO1 and SUMO2 within the cell, HEK-293 cells were co-transfected with HA-tagged PTEN together with Ubc9 and His6-tagged SUMO1, SUMO2, or pcDNA plasmids. At $48 \mathrm{~h}$ after transfection, His6-tagged proteins were purified in denaturing conditions using nickel beads. Western-blot analysis of the purified extracts with anti-HA antibody revealed bands of the expected size corresponding to PTEN-SUMO1 or PTEN-SUMO2 only in those cells cotransfected with His6-SUMO1 or His6-SUMO2, respectively, indicating that PTEN is SUMOylated in vivo (Figure 1c). To confirm that endogenous PTEN protein is also SUMOylated, protein extracts and His-tagged purified proteins obtained from HEK-293 cells transfected with His6-SUMO2 and Ubc9 were analyzed by western blot using anti-PTEN antibody. We detected an enrichment of the band of the expected size
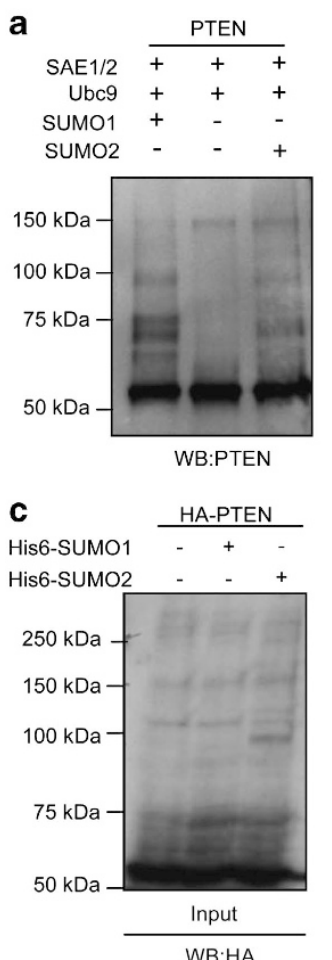
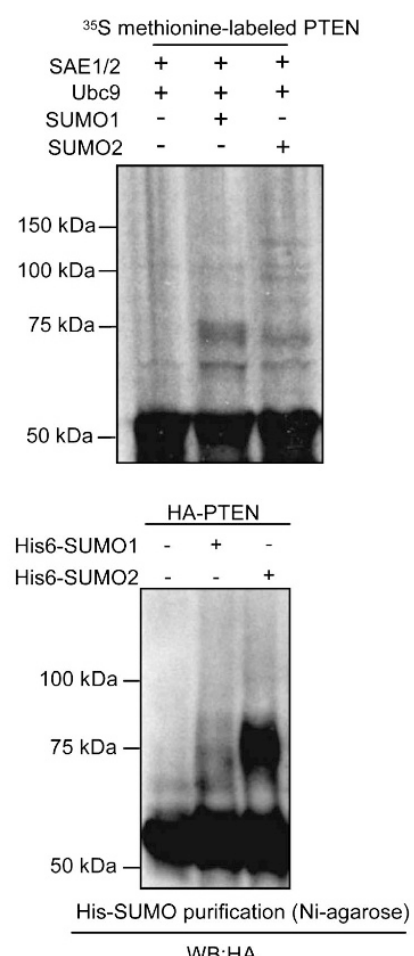

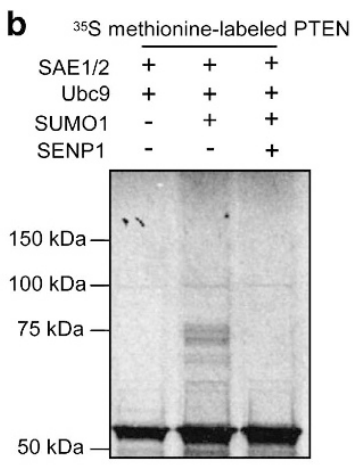

d

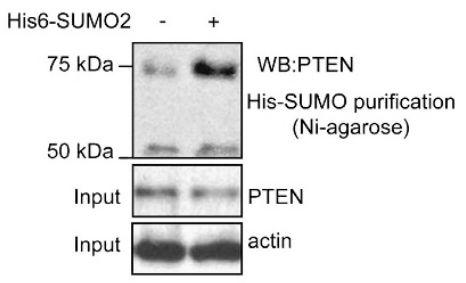

Figure 1 Covalent modification of PTEN by SUMO1 or SUMO2 in vitro and in vivo. (a) Recombinant PTEN protein (left panel) or in vitro translated ${ }^{35}$ S]methionine-labeled PTEN protein (right panel) was used as a substrate in an in vitro SUMOylation assay in the presence of SUMO1 or SUMO2. The reaction products were resolved on an $8 \%$ SDS-polyacrilamide gel and analyzed by western blot with anti-PTEN antibody (left panel) or dried for $1 \mathrm{~h}$ and exposed to X-ray film (right panel). (b) Deconjugation of SUMO1 from PTEN by SENP1. $\left[{ }^{35}\right.$ S]methionine-labeled PTEN-SUMO1 obtained in an in vitro SUMOylation reaction was incubated with GST-SENP1 as described in Materials and Methods. The reaction products were resolved on an $8 \%$ SDS-polyacrilamide gel, dried for $1 \mathrm{~h}$, and exposed to X-ray film. (c) HEK-293 cells were co-transfected with HA-PTEN together with pcDNA, pcDNA-Ubc9 and pcDNA-His6-SUMO1 or pcDNA-Ub9 and pcDNA-His6-SUMO2. Total protein extracts and the Histidine-tagged proteins purified using nickel columns were then resolved on an $8 \%$ SDS-polyacrilamide gel and analyzed by western blot with anti-HA antibody. (d) HEK-293 cells were transfected with pcDNA or pcDNA-Ubc9 and pcDNA-His6-SUMO2. Total protein extracts and the Histidine-tagged proteins purified using nickel columns were then analyzed by western blot with anti-PTEN antibody 
corresponding to PTEN-SUMO protein in the cells transfected with SUMO2 (Figure 1d). All together these data demonstrate that PTEN conjugates to SUMO1 and SUMO2 in the context of the cell. Of note, the bands corresponding to PTENSUMO1 or PTEN-SUMO2 detected in transfected cells were clearly wider than those detected after in vitro SUMOylation assays, suggesting that additional modifications may be also occurring within the cell.

Lysines $\mathbf{2 6 6}$ and $\mathbf{2 8 9}$ are SUMO-acceptor sites in PTEN. The SUMOplot prediction system identified a 252IKVE257conserved SUMOylation sequence and three more lysines as putative SUMO-conjugation residues (K102, K266, and K289). We constructed single or double PTEN mutants in the putative lysine residues and analyzed their SUMOylation in vitro. We only observed a significant reduction in SUMO1 conjugation in the single mutant in lysine residue 266, PTENK266A (Figure 2a). Interestingly, additional mutation of the lysine residue 289 further decreased the conjugation of SUMO1 to the PTENK266A mutant (Figure 2a). These results pointed to the lysine residues 266 and 289 as SUMOacceptor sites in PTEN. Then, we analyzed the conjugation of SUMO to PTEN mutants when expressed in cells. HEK293 cells were transfected with WT HA-PTEN or the indicated HA-PTEN mutants, together with Ubc9 and His6SUMO2 or pcDNA and the His6-tagged proteins were then purified and analyzed by western blot with anti-HA antibody. We detected a clear reduction in the conjugation of SUMO2 to the single PTENK266A mutant (Figure 2b). Importantly, this decrease in SUMO conjugation was most evident when we used the double PTENK266AK289A mutant as a substrate (Figure $2 b$ ). These results indicate that lysine residues 266 and 289 in PTEN are implicated in the conjugation to SUMO. Interestingly, both lysine residues, 266 and 289, localize in the C2 domain and are conserved in vertebrate PTEN (Trotman et $a l^{32}$ and Supplementary Figure S1).

PTEN mutants with reduced SUMOylation capacity are located at the cell cytoplasm. SUMO conjugation is involved in regulating the subcellular localization of different substrates. Thus, we decided to analyze the subcellular localization of the PTEN mutants that showed reduced SUMOylation. We transfected MCF-7 cells with WT HAPTEN or the PTEN SUMOylation mutants and we performed immunofluorescent staining for PTEN using anti-HA antibody. WT HA-PTEN protein was detected preferentially in the nucleus of MCF-7 cells (Figure 3a), as expected. ${ }^{33}$ Confocal analysis and quantification of at least 200 transfected cells showed a predominant cytoplasmic localization of the PTENK289A mutant, as reported previously. Similarly, both PTENK266A and the double PTENK266AK289A mutant were prevalently detected in the cell cytoplasm (Figure 3a). To verify this result, we performed western-blot analysis of the nuclear and cytoplasmic fractions of MCF-7 cells transfected with WT HA-PTEN or the HA-PTEN mutants. The results confirmed the information obtained by immunofluorescence (Figure $3 \mathrm{~b}$ ). In addition, subcellular localization of the transfected WT HA-PTEN or the HA-PTEN mutants was also analyzed in the PTEN-deficient prostate cancer cell line PC3. Confocal analysis and quantification of at least 200 transfected cells showed that WT PTEN is found mainly in the nucleus of PC3 cells $24 \mathrm{~h}$ after transfection (Figure 3c). Interestingly, at this time point all the mutants analyzed showed dominant cytoplasmic accumulation in most cells (Figure $3 \mathrm{c}$ ).
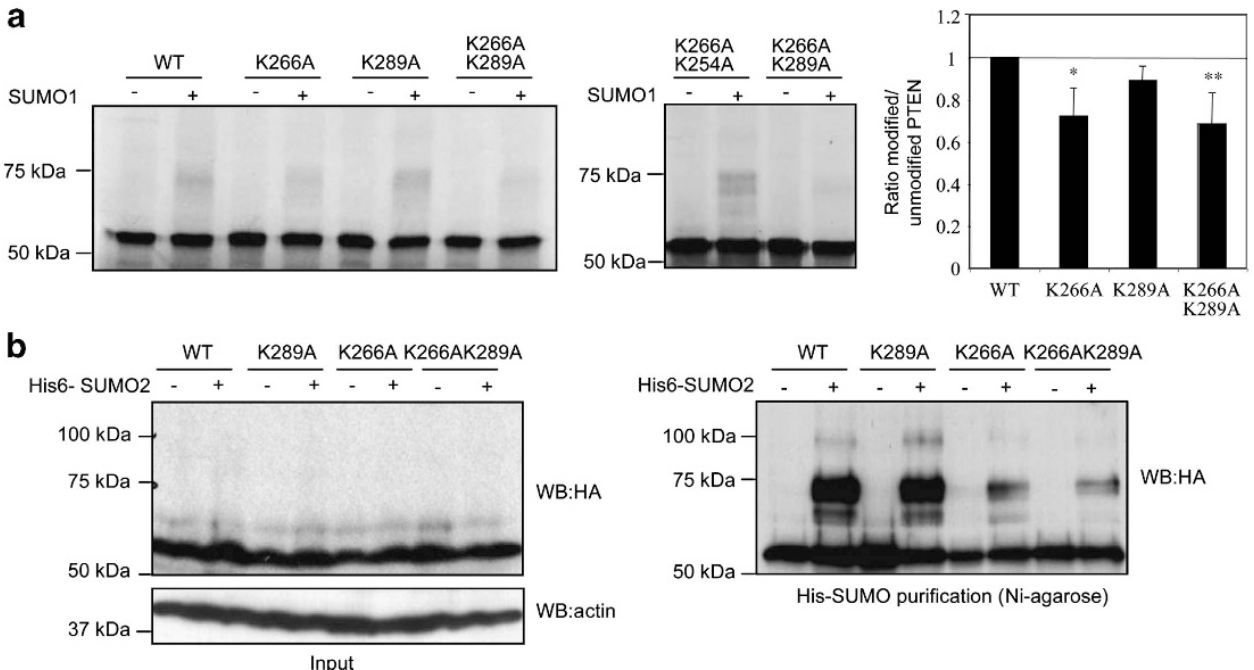

Figure 2 Identification of Lysines 266 and 289 as SUMO targets. (a) ${ }^{35}$ S]methionine-labeled wild-type or mutant PTEN proteins were used as substrates in an in vitro SUMOylation assay in the presence of SUMO1 as indicated. The reaction products were resolved on an 8\% SDS-polyacrylamide gel, dried for $1 \mathrm{~h}$, and exposed to an X-ray film. The intensity of SUMO-modified and -unmodified PTEN bands was quantified by densitometry using ImageJ software, and the ratio of SUMOylated to non-SUMOylated PTEN was normalized to that of the WT protein (right panel). The data represented in right panel are means \pm S.D. of five independent experiments. ${ }^{\star} P<0.05 ;{ }^{* \star} P<0.005$, by Student's t-test compared with PTEN WT. (b) Total protein extracts and Histidine-tagged purified proteins prepared from HEK-293 cells co-transfected with WT HA-PTEN or the different HA-PTEN mutants together with pcDNA or pcDNA-Ubc9 and pcDNA-His6-SUMO2, were resolved on an 8\% SDS-polyacrilamide gel and analyzed by western blot with anti-HA antibody 

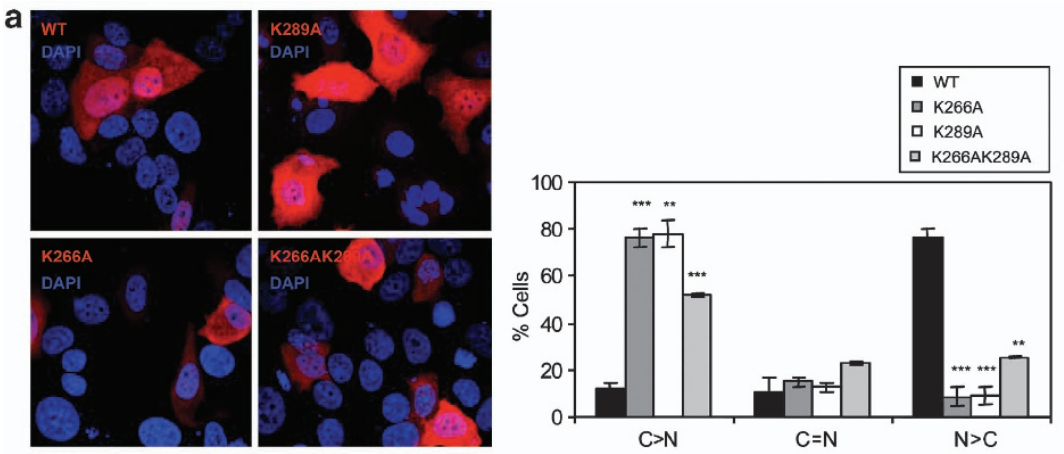

b
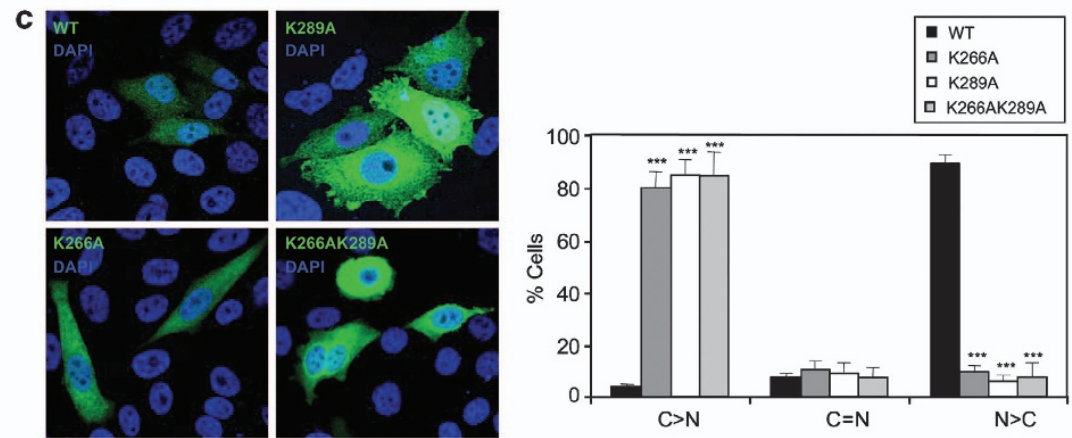

Figure 3 Cytoplasmic localization of SUMOylation-deficient PTEN mutants. (a) MCF-7 cells were transfected with plasmids encoding WT or mutant HA-PTEN proteins and then stained with anti-HA antibody followed by Alexa 594-conjugated anti-mouse antibody and DAPI. Subcellular localization of the expressed proteins was analyzed under a confocal laser scanning microscope. Images were processed with Adobe Photoshop. Right panel shows quantification of the subcellular distribution of PTEN in at least 200 transfected cells per sample. (b) MCF-7 cells were transfected as described above and at $48 \mathrm{~h}$ after transfection, nuclear and cytoplasmic fractions were isolated, resolved on an $12 \%$ SDS-polyacrylamide gel and analyzed by western blot with the indicated antibodies. (c) PC3 cells were transfected with plasmids encoding WT or mutant HA-PTEN proteins, then stained with anti-PTEN antibody followed by Alexa 488-conjugated anti-mouse antibody and DAPI, and finally analyzed by confocal microscopy. Right panel shows quantification of the subcellular distribution of PTEN in at least 200 transfected cells per sample. ${ }^{* \star} P<0.005,{ }^{* \star *} P<0.0005$, by Student's $t$-test compared with PTEN WT

Interplay between SUMOylation and ubiquitination of PTEN. Previously, it has been demonstrated that lysine 289 is a major site for PTEN monoubiquitination and that this modification leads to the nuclear translocation of PTEN. ${ }^{32}$ In addition, we have shown that a mutant in lysine 266 was mainly detected in the cell cytoplasm, raising the possibility that this residue could also be a site for monoubiquitin conjugation in PTEN. To evaluate this possibility, we decided to study the effects of mutating lysine residue 266 on the monoubiquitination of PTEN by performing ubiquitination in vitro using the ubiquitin $\mathrm{K}$ all $\mathrm{R}$ mutant (Ub-KO), in which each lysine residue in ubiquitin is replaced by an arginine, blocking the formation of polyubiquitin chains. We observed a slight but reproducibly significant reduction in the Ub-KO conjugation to PTENK266A, PTENK289A or PTENK266AK289A mutant (Figure 4a), indicating that both lysine residues, 266 and 289, are susceptible to conjugate ubiquitin.

Our results indicated that the lysine residues 266 and 289 are implicated in the conjugation to SUMO, and that the lysine residue 266 , in addition to the already known monoubiquitination of lysine 289 , is also susceptible to conjugate monoubiquitin. Based on these data, we hypothesized that PTEN SUMOylation could modulate the covalent interaction of PTEN with monoubiquitin. To evaluate this possibility, we carried out an in vitro monoubiquitination assay using recombinant PTEN or PTEN-SUMO1 as a substrate and then we analyzed the modified proteins by western blot using antiPTEN or anti-ubiquitin antibodies. As shown in Figure 4b, we noticed that the anti-ubiquitin antibody recognized monoubiquitin-PTEN protein but anti-PTEN antibody did not. PTENSUMO1 bands detected using anti-PTEN antibody were similar independently of its subsequent incubation with monoubiquitin. In addition, the smear of monoubiquitinated PTEN detected when we used PTEN as a substrate was clearly reduced when we employed PTEN-SUMO1 as a substrate (Figure $4 b$ ). These results indicate that conjugation of SUMO1 to PTEN reduces its conjugation with monoubiquitin.

It has been also shown that, upon inhibition of the proteasome, the amount of SUMO2 conjugates of a large set of target proteins accumulate. ${ }^{23}$ We then decided to evaluate whether PTEN is one of these substrates. HEK-293 cells were co-transfected with HA-PTEN together with pcDNA or pcDNA-His6-SUMO2, and Ubc9, and then treated with the proteasome inhibitor MG132. His6-SUMO2-PTEN conjugates were purified, and immunoblot experiments using anti$\mathrm{HA}$, anti-SUMO2 or anti-ubiquitin antibodies were carried out. As shown in Figure 4c, we detected the accumulation of PTEN-SUMO2-purified conjugates in those cells treated with 

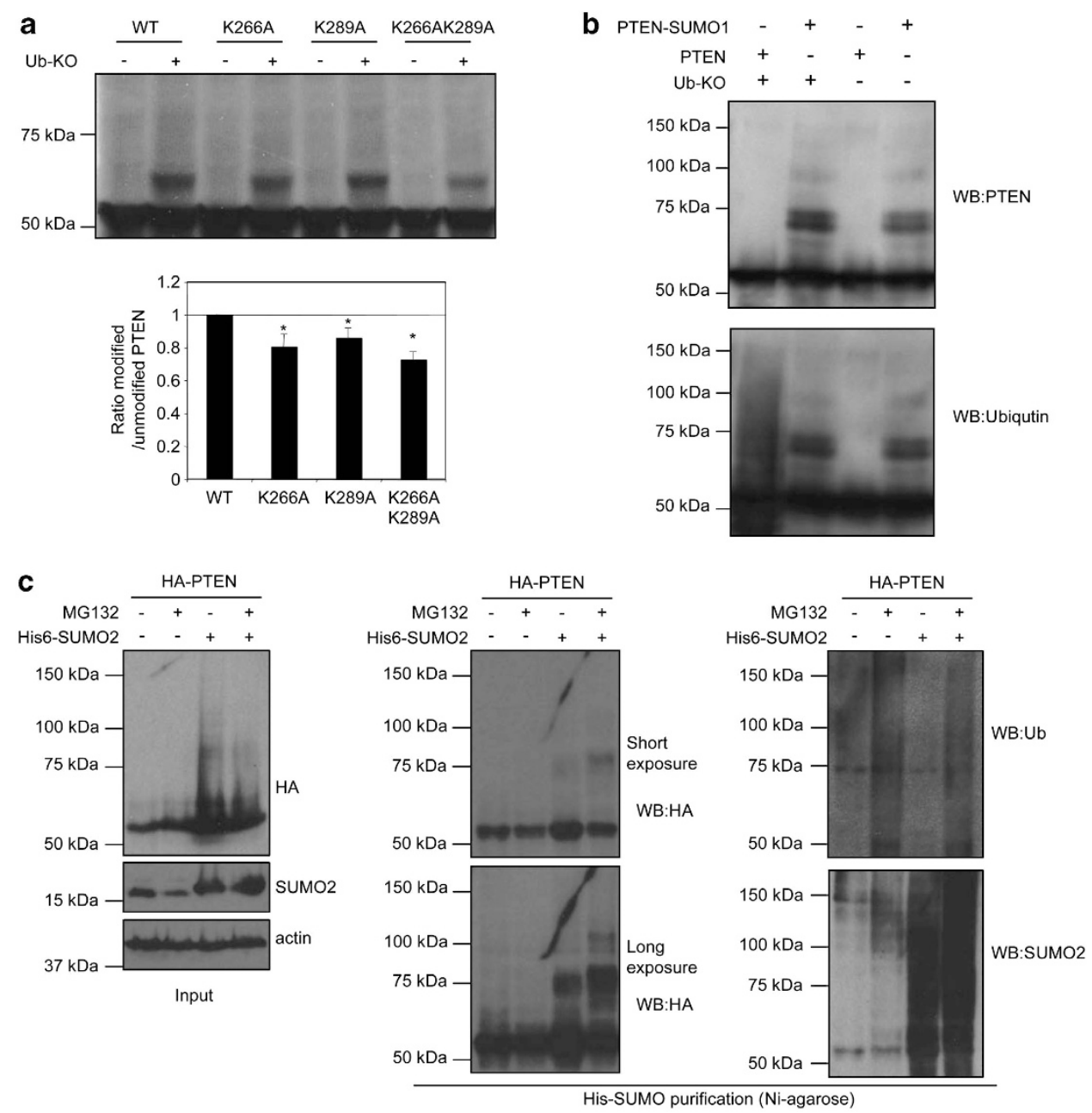

Figure 4 Crosstalk between SUMOylation and ubiquitination of PTEN. (a) $\left[{ }^{35}\right.$ S $]$ methionine-labeled wild-type or mutant PTEN proteins were used as substrates in an in vitro ubiquitination assay in the presence of $\mathrm{Ub}-\mathrm{KO}$ as indicated. The reaction products were resolved on an $8 \%$ SDS-polyacrylamide gel, dried for $1 \mathrm{~h}$, and exposed to an $X$-ray film. The intensity of ubiquitin-modified and -unmodified PTEN bands was quantified by densitometry using ImageJ software, and the ratio of ubiquitinated to nonubiquitinated PTEN was normalized to that of the WT protein (lower panel). The data represented are means \pm S.D. of at least three independent experiments. ${ }^{\star} P<0.05$, by Student's t-test compared with PTEN WT. (b) Recombinant PTEN protein was used as a substrate in an in vitro SUMOylation assay in the presence or absence of SUMO1 and then the products of the SUMOylation reactions were used as substrates in an in vitro ubiquitination assay as indicated. The reaction products were then resolved on an $8 \%$ SDS-polyacrylamide gel and analyzed by western blot with anti-PTEN antibody. Membranes were subsequently washed and then incubated with anti-ubiquitin antibody. (c) HEK-293 cells were co-transfected with HA-PTEN together with pcDNA or Ubc9 and His6-SUMO2 and at $24 \mathrm{~h}$ after transfection cells were treated with MG132 (25 mM) for $12 \mathrm{~h}$. Total protein extracts and the Histidine-tagged proteins purified using nickel columns were then resolved on an $8 \%$ SDS-polyacrylamide gel and analyzed by western blot with the indicated antibodies. Ub, ubiquitin

MG132, suggesting that conjugation of SUMO2 to PTEN might positively modulate its covalent interaction with ubiquitin.

Vesicular stomatitis virus (VSV) infection induces SUMOylation of PTEN and its translocation to the cell membrane. There is evidence that virus infection can alter the SUMOylation status of host cell proteins ${ }^{34}$ and results from our laboratory suggested that PTEN was one of these altered cellular proteins. To study this possibility, we infected mouse embryo fibroblasts (MEFs) derived from WT mice with VSV at a MOI of $5 \mathrm{PFU} / \mathrm{ml}$ and at different times after infection, we analyzed the PTEN protein by western blot. As shown in Figure 5a, we did not detect significant changes in PTEN protein levels at any time. However, we detected an additional band of $\sim 70-75 \mathrm{kDa}$ only in those cells infected with VSV (Figure $5 \mathrm{a}$, left panel). A similar additional band was also detected by western blot analysis of cells transfected with HA-PTEN and infected with VSV using an anti-HA antibody (Figure 5a, right panel). These results suggested that VSV infection induced a post-translational modification of PTEN and we speculated that this modification might correspond with our previously identified SUMO conjugation. To evaluate this hypothesis, we analyzed PTEN SUMOylation in uninfected cells and at different times after VSV infection. HEK-293 cells were co-transfected with HA-PTEN, Ubc9, and His6-SUMO2, and after $24 \mathrm{~h}$, cells were infected with VSV and at different times after infection, His6-tagged proteins were purified, and analyzed with anti-HA antibody. We detected PTEN-SUMO2 bands in those cells 
a

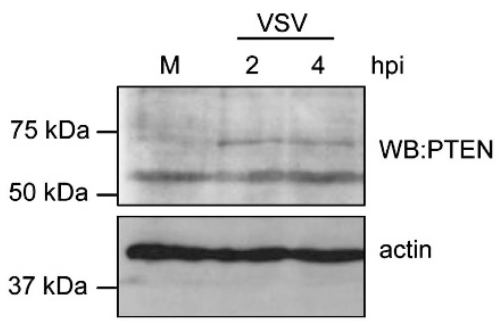

b
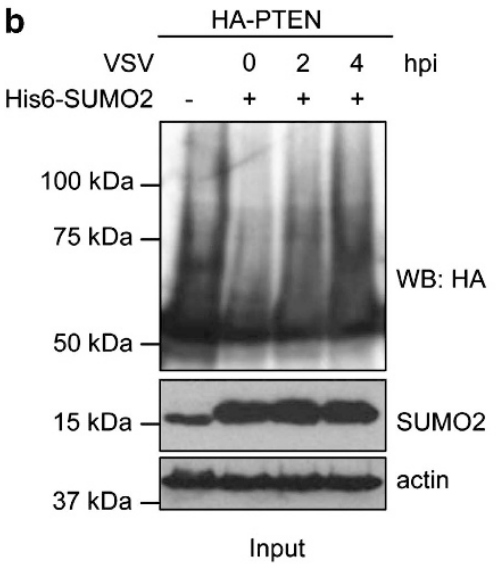
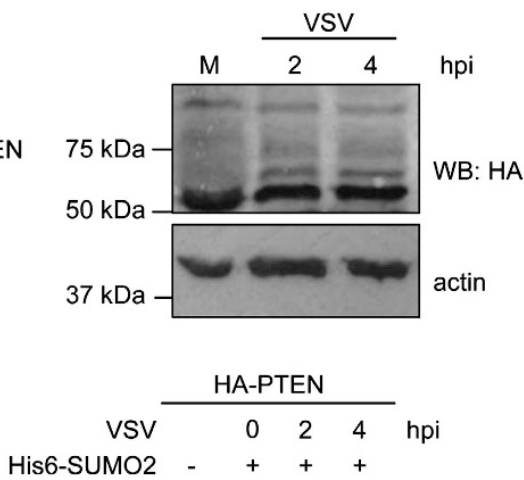

His6-SUMO2 - +++

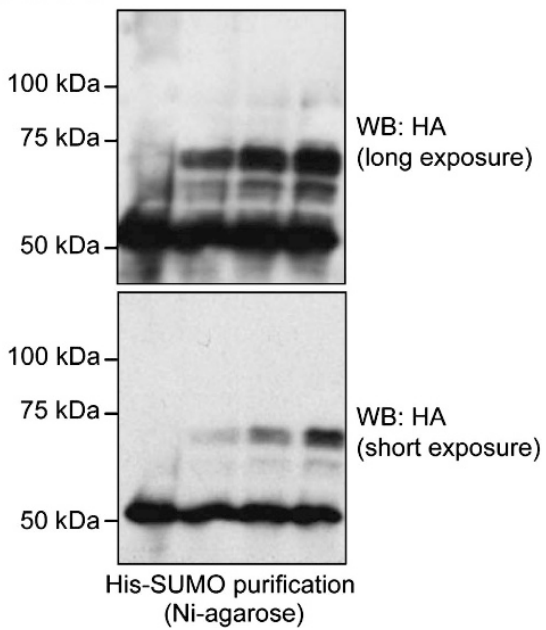

Figure 5 VSV infection promotes SUMOylation of PTEN. (a) MEFs WT (left panel) or MCF-7 cells transfected with HA-PTEN (right panel) were left uninfected (U) or infected with VSV for the indicated times and then cell lysates were resolved on an $8 \%$ SDS-polyacrylamide gel, and analyzed by western blot with anti-PTEN (left panel) or anti-HA antibody (right panel). (b) HEK-293 cells were co-transfected with HA-PTEN together with pcDNA, pcDNA-Ubc9 and pcDNA-His6-SUMO2 and $24 \mathrm{~h}$ after transfection cells were infected with VSV at MOI of $5 \mathrm{PFU} / \mathrm{ml}$. Total protein extracts and the Histidine-tagged proteins purified using nickel columns at the indicated times after infection were resolved on an $8 \%$ SDS-polyacrylamide gel and analyzed by western blot with the indicated antibodies

transfected with HA-PTEN, Ubc9, and His6-SUMO2 (Figure $5 \mathrm{~b}$ ), as expected. The amount of PTEN-SUMO2conjugated protein clearly increased in those cells infected with VSV in a time-dependent manner (Figure 5b), indicating that VSV infection stimulates PTEN SUMOylation. Then, we decided to analyze the subcellular localization of PTEN in cells infected with VSV. HA-PTEN transfected U251MG cells, and MEFs, were infected with VSV at a MOI of 5 , and $4 \mathrm{~h}$ after infection cells were immunostained with anti-HA or anti-PTEN antibodies, respectively. Confocal analysis and quantification of at least 200 cells revealed that PTEN was localized at the plasma membrane in $45 \%$ of the infected cells, whereas only $2 \%$ of the non-infected cells showed PTEN at this subcellular localization (Figure 6a). Similarly, we also detected the translocation of HA-PTEN to the plasma membrane in 39\% U251MG cells infected with VSV in comparison with $3 \%$ of the non-infected cells (Figure $6 \mathrm{~b}$ ). These data suggested that, in response to VSV infection, a specific pool of PTEN moves to the cell membrane and is post-translationally modified. Thus, we decided to analyze the putative co-localization of SUMO and PTEN in the plasma membrane of the infected cells. MEFs uninfected, or at $4 \mathrm{~h}$ after infection with VSV, were immunostained with both
anti-PTEN, and anti-SUMO1 or anti-SUMO2 antibodies. Confocal analysis revealed that both, PTEN and SUMO1 or SUMO2, were mainly detected in the cell nucleus of the uninfected cells (only $4 \%$ of the non-infected cells showed PTEN and SUMO1 or SUMO2 co-localizing at the cell membrane), whereas $42 \%$ and $40 \%$ of the VSV infected cells showed co-localization between PTEN and SUMO1 or SUMO2 at the cell membrane, respectively (Figure 6c). All together, these data indicate that VSV infection induces the SUMOylation of PTEN and its translocation to the cell membrane.

SUMOylation of PTEN contributes to the control of VSV. It has been previously demonstrated that replication of VSV in mice and Drosophila can be modulated by PTEN. ${ }^{13,15}$ Thus, we decided to evaluate the consequences of PTEN SUMOylation on the replication of the virus. First, we analyzed whether VSV replication in PTEN-null U251MG cells was affected by the expression of PTEN. We infected U251MG cells, previously transfected with PML-IV, as a positive control, HA-PTEN or an empty vector, with VSV at a $\mathrm{MOI}$ of $5 \mathrm{PFU} / \mathrm{ml}$ and virus titers in the supernatants, after all cells died as a result of the infection, were determined. 
a
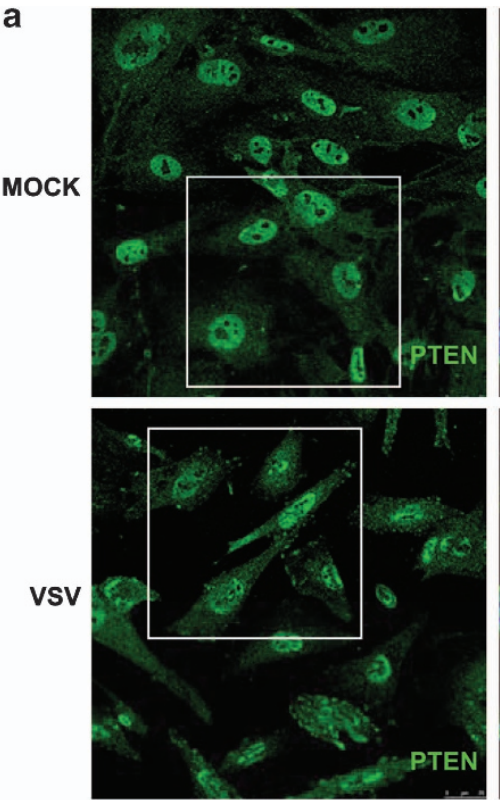

b
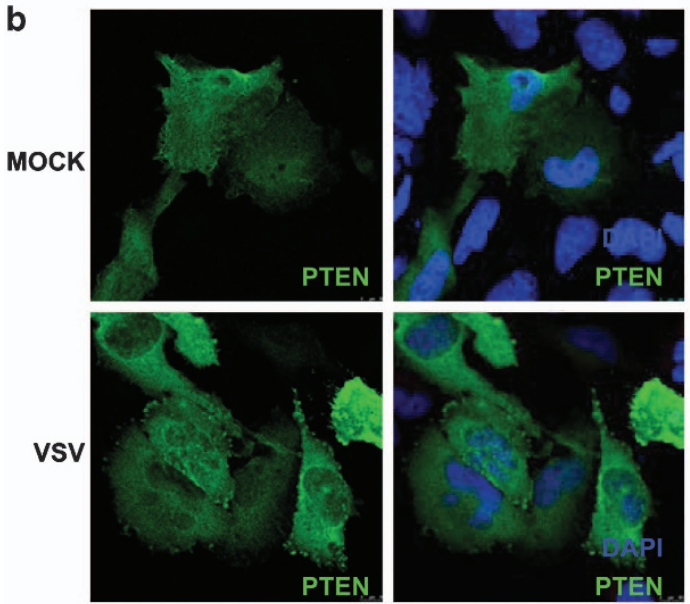
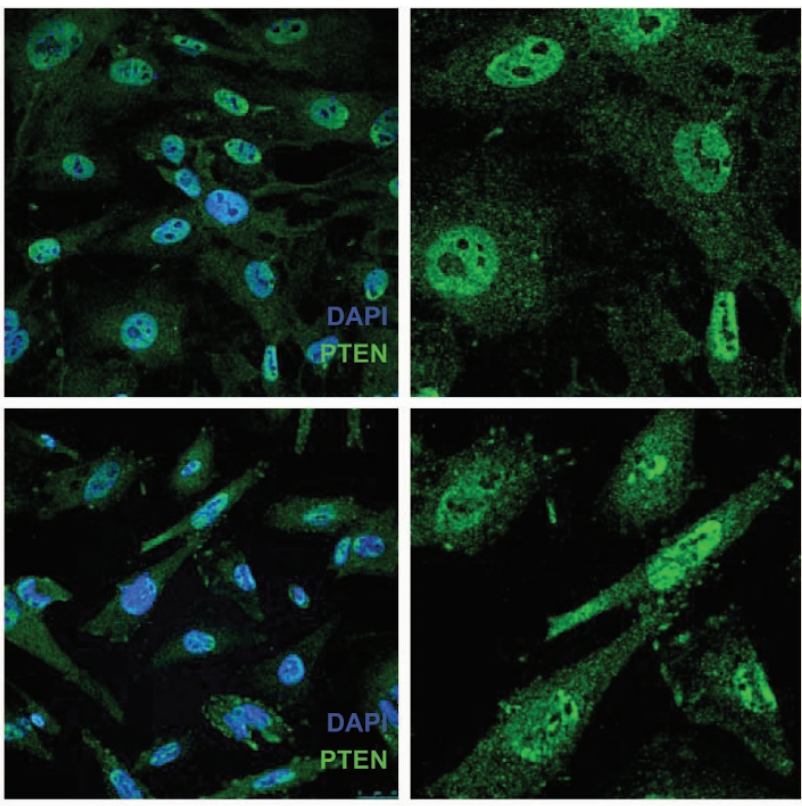

C
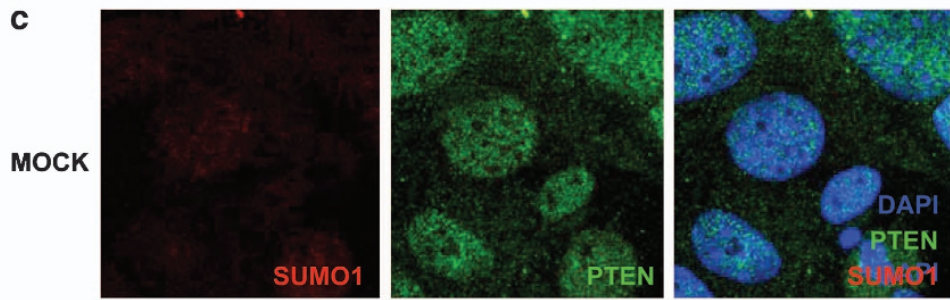
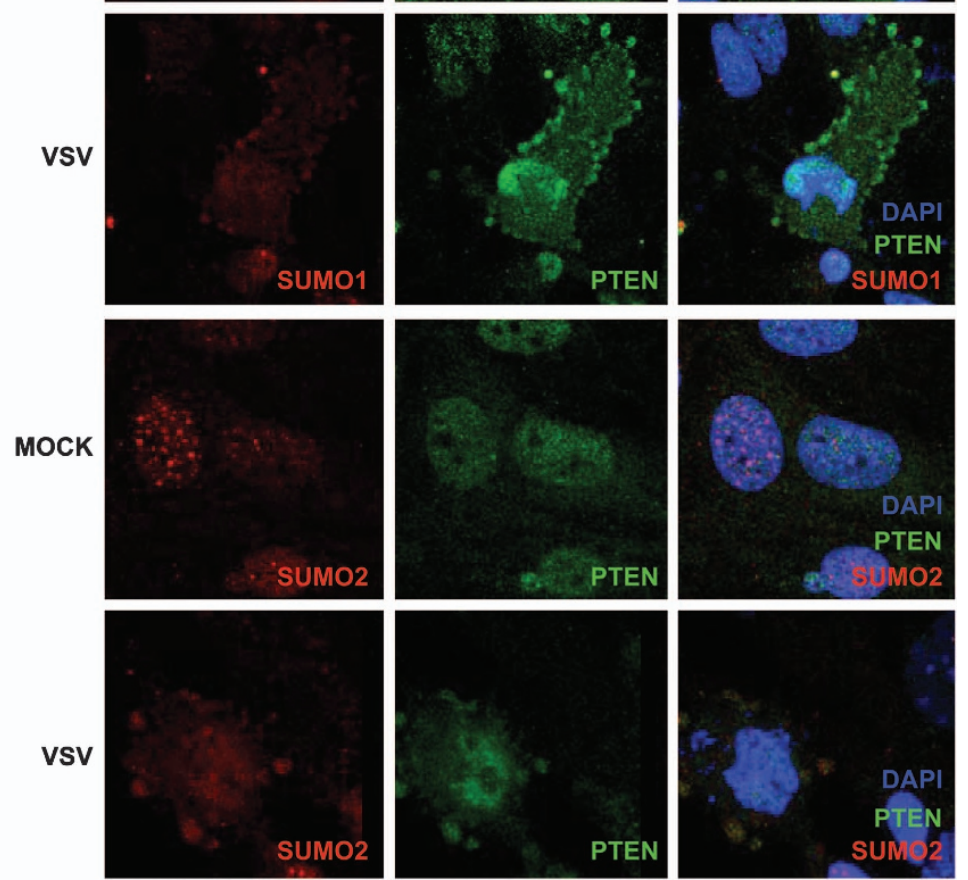

Figure 6 VSV infection promotes membrane localization of PTEN. (a) MEFs WT uninfected or infected with VSV for $4 \mathrm{~h}$ were analyzed by immunofluorescence staining for subcellular localization of PTEN. Cells were stained with anti-PTEN antibody followed by Alexa 488-conjugated anti-mouse antibody and DAPI, and finally analyzed by confocal microscopy. Digital zoom $(\times 2)$ of the indicated area is shown in the right panels. (b) PTEN-null U251MG cells transfected with HA-PTEN, uninfected or infected with VSV for $4 \mathrm{~h}$ were analyzed by immunofluorescence staining for subcellular localization of PTEN. Cells were stained with anti-HA antibody followed by Alexa 488-conjugated anti-mouse antibody and DAPI, and finally analyzed by confocal microscopy. (c) Co-localization of PTEN and SUMO at the plasma membrane. MEFs were infected with VSV, fixed and stained with anti-PTEN and anti-SUMO1 or anti-SUMO2 antibodies and DAPI. The images were analyzed by confocal microscopy 
a
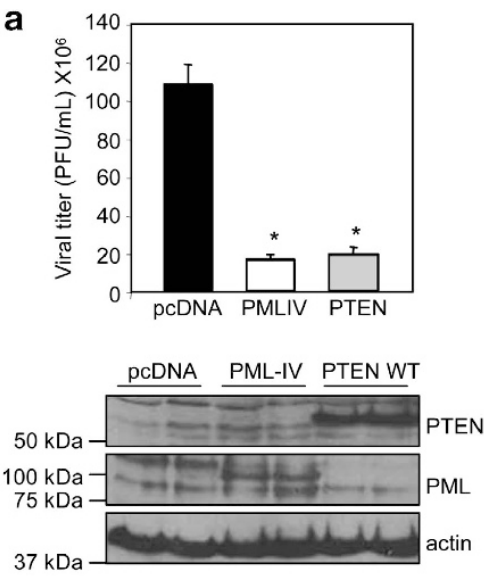

b

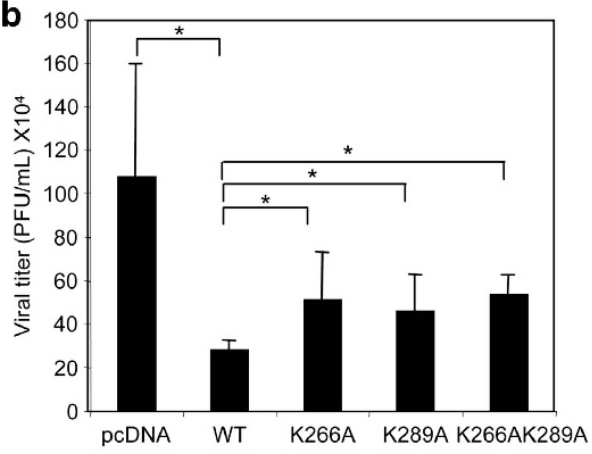

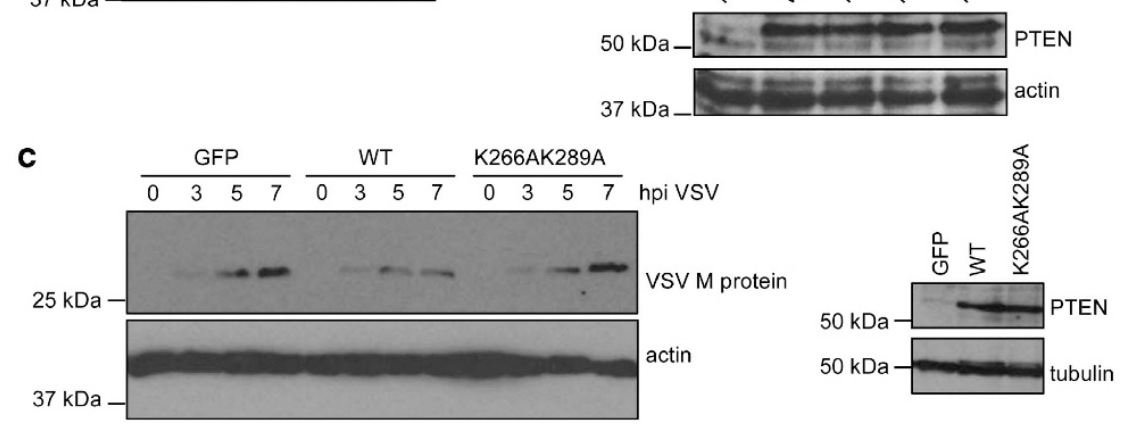

Figure 7 Effect of PTEN WT or PTEN SUMOylation mutants on VSV replication. (a) U251MG cells were transfected in triplicate with the indicated plasmids and at $48 \mathrm{~h}$ after transfection, cells were infected with VSV at MOI of 5 and quantification of the virus yield after total destruction of the monolayer was assessed. Data represents means \pm S.E. for one experiment. ${ }^{*} P<0.05$, compared with pcDNA transfected cells, Student's $t$-test. Protein extracts from the transfected cells were loaded on an $8 \%$ SDSpolyacrylamide gel and revealed on western blot by the indicated antibodies. The lower panel represents the expression levels of PML-IV (positive control) and PTEN genes transfected in two wells of one experiment. (b) U251MG cells were transfected in triplicate with the indicated plasmids and at $48 \mathrm{~h}$ after transfection cells were infected with VSV at MOI of 5 and quantification of the virus yield after total destruction of the monolayer was assessed. Data represents means $\pm S$.E. for one experiment. ${ }^{*} P<0.05$, Student's $t$-test. Protein extracts from the transfected cells were loaded on an $8 \%$ SDS-polyacrylamide gel and revealed on western blot by PTEN antibody. (c) PTEN ${ }^{+1-}$ MEFs were nucleofected with the indicated plasmids and at $48 \mathrm{~h}$ after transfection, cells were infected with VSV at MOI of 5 . At the indicated times, protein extracts were loaded on a $12 \%$ SDS-polyacrylamide gel and revealed on western blot by antibodies against the M protein from VSV. The right panel represents the expression levels of PTEN genes transfected in the cells

We detected a decrease in the number of infectious viral particles produced in U251MG cells transfected with PML in comparison with the number detected in the supernatant of pcDNA transfected cells (Figure 7a), as expected. ${ }^{35}$ In addition, we observed that expression of PTEN resulted in a similar decrease in the viral titer (Figure 7a), indicating that PTEN expression inhibits VSV replication in U251MG cells. Then, we analyzed the replication of VSV in U251MG cells transfected with WT PTEN or the PTEN SUMOylation mutants. We observed a statistically significant reduction in the VSV titer recovered from U251MG cells transfected with WT PTEN in comparison with the titer observed in pcDNA transfected cells (Figure $7 \mathrm{~b}$ ), reinforcing the data shown above. Expression of the PTEN SUMOylation mutants partially reduced the antiviral effect mediated by the WT protein (Figure 7b). Finally we evaluated the effect of WT PTEN or the PTENK266AK289A mutant on VSV protein synthesis. We detected a decrease in the levels of VSV M protein in those PTEN \pm MEFs nucleofected with WT PTEN that was not observed after expression of the PTEN SUMOylation mutant (Figure 7c). All together these results indicate that SUMOylation of PTEN is a mechanism that contributes to the cellular antiviral response.

\section{Discussion}

Here we have demonstrated that PTEN is conjugated to both, SUMO1 and SUMO2. Covalent interaction of PTEN with SUMO1 in vitro led to the formation of PTEN-SUMO1 conjugates with different molecular weights, indicating that different lysine residues in PTEN are susceptible to conjugate SUMO. The analysis of several PTEN mutants using in vitro and in vivo SUMOylation assays led us to identify lysine residues 266 and 289 as SUMO acceptors in PTEN. However, SUMOylation was not totally absent, indicating the presence of additional non-consensus site for SUMO modification. Lysine 289 has been previously identified as a major monoubiquitin acceptor in PTEN. ${ }^{32}$ Our results also showed that lysine residue 266 is part of the set of ubiquitin target lysines of PTEN, suggesting that SUMO and ubiquitin might counteract each other by competing for the same acceptor lysine, as it occurs with IkBa or PCNA. ${ }^{24,25,27}$ The role of 
monoubiquitination in promoting the nuclear localization of $\mathrm{PTEN}^{36}$ might explain the cytoplasmic localization of the double SUMOylation and monoubiquitination-deficient PTEN proteins. We also observed a defect in the monoubiquitin conjugation to PTEN-SUMO1 complexes using in vitro assays. However, there are other situations in which SUMO and ubiquitin pathways can intersect. In this sense, it has been recently demonstrated that there are SUMO-targeted ubiquitin ligases, which ubiquitinate and consequently target SUMO-modified proteins to the proteasome for degradation. $^{28,29}$ Here we demonstrated that PTEN is SUMO2 modified in a proteasome inhibitor-sensitive manner, as reported also for other substrates. ${ }^{23}$ The reason for the PTEN-SUMO2 or SUMO2-ubiquitin chains accumulation after proteasome inhibition is unknown. One possibility would be that the protein is targeted to the proteasome via these chains, as reported for $\mathrm{PML}^{28,29}$ or that these chains regulate degradation-independent processes.

The SUMOylation system can be regulated through multiple mechanisms, including the modulation of the expression of various components of the SUMOylation pathway, and the regulation of the activity of SUMO enzymes. In addition, SUMOylation of a specific protein can be influenced by its cellular localization, the presence of specific stimuli, or the availability and activity of SUMO conjugation enzymes. In this sense, different stresses, such as heat shock and osmotic stress can increase SUMOylation of different proteins in cells in culture, whereas moderate levels of oxidative stress inactivate SUMO conjugation enzymes. Viruses can also work as a stimulus that lead to the deregulation of the SUMO pathway, ${ }^{37,38}$ downmodulate the levels of specific SUMOylated proteins ${ }^{39,40}$ or induce the SUMOylation of cellular proteins. ${ }^{41-45}$ Our results demonstrate that VSV infection induces PTEN SUMOylation. In parallel to its SUMOylation, VSV infection induces the translocation of PTEN to the plasma membrane, where it co-localizes with SUMO1 or SUMO2, suggesting that both PTEN SUMOylation and membrane localization could be related. The localization of PTEN at the plasma membrane requires the $\mathrm{C} 2$ domain and is negatively regulated by a phosphorylation-dependent intramolecular interaction. ${ }^{46}$ The phoshorylated C-terminal domain of PTEN associates with the $\mathrm{C} 2$ domain in the $\mathrm{N}$-terminus of the protein in a closed conformation, negatively regulating the interaction of PTEN with the plasma membrane, while an 'open' conformation of PTEN favors such interaction. ${ }^{46}$ Based on this model and our results, we hypothesize that the covalent interaction of SUMO with lysine residues within the $\mathrm{C} 2$ domain of PTEN may negatively regulate its association with the $\mathrm{C}$-terminal fragment of the protein, maintaining PTEN in an 'open' conformation, and favoring the interaction between PTEN and the plasma membrane.

SUMOylation has been involved in multiple vital cellular processes, such as oncogenesis, cell cycle control, nucleocytoplasmic traffic, apoptosis and response to virus infection. ${ }^{20,24,34,47-49}$ There are some reports that suggest that PTEN may have a role in the antiviral response. Viral proteins that contribute to the pathogenesis of human immunodeficiency virus type-1, simian immunodeficiency virus or Hepatitis B virus, downmodulate the levels of PTEN protein. ${ }^{50,51}$ Furthermore, the replication of VSV was modulated in a PTEN-dependent manner in both Drosophila and mice. ${ }^{13,15}$ In addition, it has been extensively reported that VSV infection rapidly induces an antiviral state in the host cell, as exemplified by the induction of an interferon response. ${ }^{52-54}$ Thus, we hypothesized that the induction of PTEN SUMOylation by VSV infection may have a role in the antiviral response. To address this issue, we evaluated how PTEN SUMOylation affects VSV replication. Our results show that VSV replication is reduced after PTEN WT reintroduction in PTEN-null U251MG cells, but not when the PTEN SUMOylation mutants were used, indicating that this pathway has a role in the antiviral response. Hence, the induction of PTEN SUMOylation by VSV may be considered as a means to boost a cellular response to virus replication.

In summary, our data demonstrate that PTEN is modified by SUMO1 and SUMO2, and reveal the existence of a crosstalk between SUMOylation and ubiquitination of PTEN. In addition, our work demonstrates that VSV infection stimulates PTEN SUMOylation and its localization at the cell membrane, a previously unknown mechanism for modulating virus replication. Finally, we propose that SUMOylation may work as a mechanism to inhibit the intramolecular interaction in PTEN and to increase PTEN membrane association. The modification of PTEN by SUMO1 as a mechanism controlling its association with the plasma membrane has been published recently while our manuscript was under review, ${ }^{55}$ further supporting our results. The identification of VSV infection as a mechanism modulating PTEN post-translational modification provides a novel means of controlling PTEN function and offers new ideas for the development of new therapies for PTEN-associated diseases.

\section{Materials and Methods}

Cell lines and transfections. MEFs derived from heterozygous PTEN $\left(\mathrm{PTEN}^{+l-}\right)^{56}$ or WT mice, and the cell lines MCF-7, HEK-293, BSC-40, U251MG, and PC3 were maintained in DMEM supplemented with $10 \%$ heatinactivated-fetal calf serum (Gibco, Life Technologies SA, Madrid, Spain), $5 \mathrm{mmol} / \mathrm{I}$ L-glutamine (Invitrogen, Life Technologies SA, Madrid, Spain) and 1\% penicillin-streptomycin solution (Sigma-Aldrich Quimica SA, Madrid, Spain, $10000 \mathrm{U} / \mathrm{ml}$ penicillin and $10 \mathrm{mg} / \mathrm{ml}$ streptomycin). Transfection of HEK-293 and PC3 was done using X-treme (Roche Farma, SA, Madrid, Spain), MCF-7 cells were transfected with X-treme-HD and MEFs were transfected using the Amaxa Biosystems (Lonza, Porriño, Spain) nucleofection system, following the manufacturer's instructions. Infections were carried out using VSV of Indiana strain and virus yields were measured by plaque assays in BSC-40 cells.

Plasmids and reagents. HA-PTEN plasmid has been previously described. ${ }^{57}$ The GFP plasmid was purchased from Clontech (Saint-Germainen-Laye, France). All site-directed mutagenesis were carried out using the QuickChange polymerase chain reaction-based mutagenesis kit (Stratagene, La Jolla, CA, USA), and the HA-PTEN plasmid as a template. Oligonucleotides used in the site-directed mutagenesis are listed in Supplementary Table 1. pcDNA-His6SUMO1, pcDNA-His6-SUMO2, and pcDNA-Ubc9 have been previously described. ${ }^{25,58}$

In vitro SUMO conjugation assay. In vitro SUMO conjugation assay was performed on recombinant PTEN protein (Upstate, Izasa SA, Barcelona, Spain) or ${ }^{35}$ S]methionine-labeled in vitro transcribed/translated PTEN proteins as described previously ${ }^{59}$ using recombinant E1 (Biomol, Enzo Life Sciences, Lausen, Switzerland), Ubc9, and SUMO1 or SUMO2. Reaction products were fractionated on an $8 \%$ SDS-polyacrylamide gel. Recombinant PTEN protein was analyzed using western-blot analysis with anti-PTEN antibody and $\left[{ }^{35}\right.$ S]methionine-labeled 
in vitro transcribed/translated PTEN protein was detected by autoradiography. In vitro transcription/translation of proteins was performed using $1 \mu \mathrm{g}$ of plasmid DNA and rabbit reticulocyte-coupled transcription/translation system according to the instructions provided by the manufacturer (Promega Biotech Iberica, SL, Madrid, Spain).

In vitro deSUMOylation assay. PTEN-SUMO1 obtained in an in vitro SUMOylation reaction as described above was incubated with $2 \mu \mathrm{g}$ of GSTSENP1 (Biomol) in $30 \mu \mathrm{l}$ reaction buffer containing $50 \mathrm{mM}$ Tris $(\mathrm{pH} 7.5), 2 \mathrm{mM}$ $\mathrm{MgCl}_{2}$, and $5 \mathrm{mM} \beta$-mercaptoethanol. Reactions were incubated at $37^{\circ} \mathrm{C}$ for $1 \mathrm{~h}$ and terminated with SDS sample buffer containing mercaptoethanol. Reactions products were then fractionated on an $8 \%$ SDS-polyacrylamide gel, dried for $1 \mathrm{~h}$, and exposed to X-ray film.

In vitro ubiquitylation assay. $\left[{ }^{35} \mathrm{~S}\right]$ methionine-labeled in vitro transcribed/ translated PTEN was incubated in a $10 \mu$ l reaction including an ATP regenerating system ( $50 \mathrm{mM}$ Tris pH 7.6, $5 \mathrm{mM} \mathrm{MgCl2,} 2 \mathrm{mM}$ ATP, $10 \mathrm{mM}$ creatine phosphate, $3.5 \mathrm{U} / \mathrm{ml}$ of creatine kinase and $0.6 \mathrm{U} / \mathrm{ml}$ of inorganic pyrophosphatase), $10 \mathrm{ng}$ human $\mathrm{E} 1,12 \mathrm{ng} \mathrm{E} 2$, and $10 \mu \mathrm{g}$ of a ubiquitin mutant in which each lysine residue within ubiquitin is mutated to arginine (referred to as ubiquitin-KO) to block the formation of polyubiquitin chains. After incubation at $30^{\circ} \mathrm{C}$ for $45 \mathrm{~min}$, the reaction products were treated as described for the SUMO conjugation assay.

Western-blot analysis and antibodies. For western-blot analysis, cells were washed in PBS, scraped in SDS gel-loading buffer and boiled for $5 \mathrm{~min}$. Proteins of total extracts were separated by SDS-PAGE and transferred to nitrocellulose membrane. The following antibodies were used: anti-SUMO1 (sc-9060, Santa Cruz Biotechnology, Santa Cruz, CA, USA), anti-PML (sc-5621, Santa Cruz), anti-SUMO2 (51-9100, Zymed Laboratories, Life Technologies SA, Madrid, Spain), anti-actin (69100, MP Biomedicals, Europe, Illkirch, France), antiB23 (sc-5564, Santa Cruz), anti-HA (MMS-101P, Covance, Inc., Madrid, Spain), anti-PTEN (9556, Cell Signaling, Izasa SA, Barcelona, Spain), anti-ubiquitin (sc-8017, Santa Cruz), anti-VSV M protein (EB0011, KeraFAST), anti-tubulin (MCA78G, AbD serotec, Düsseldorf, Germany), anti-GAPDH (MAB374, Millipore Iberica SAU, Madrid, Spain), Alexa 488-conjugated anti-mouse antibody (A-11029, Molecular Probes, Life Technologies SA, Madrid, Spain), Alexa 594-conjugated anti-mouse antibody (A-11005, Molecular Probes), and Alexa 594-conjugated antirabbit antibody (A-11037, Molecular Probes).

Immunofluorescence staining. Immunofluorescence staining and confocal analysis were performed as described. ${ }^{59}$ Analysis of the samples was carried out on a Leica TCS SP5 confocal laser microscope (Leica Microsistemas SLU, Barcelona, Spain) using simultaneous scans to avoid shift between the optical channels. Images were exported using Adobe Photoshop CS2 version 9.0.2 (Hewlett Packard Company, Boise, ID, USA).

Purification of His-tagged conjugates. Purification of His-tagged conjugates using $\mathrm{Ni}^{2+}$-NTA-agarose beads was performed as described. ${ }^{60}$

Statistical analysis. For statistical analysis between control and different groups the Student's $t$-test was applied. The significance level chosen for the statistical analysis was $P<0.05$.

\section{Conflict of Interest}

The authors declare no conflict of interest.

Acknowledgements. We thank Manuel Collado for valuable criticism of the manuscript. Funding at the laboratory of CR is provided by BFU-2011-27064. MC and LMV are supported by Juan de la Cierva Program. JG-S is supported by an IFARHU-SENACYT predoctoral fellowship from Panama. PG is supported by JAE predoctoral fellowship from CSIC. CFC-H is supported by La Caixa fellowship. The funders had no role in the study design, data collection and analysis, decision to publish, or preparation of the manuscript.

1. Li DM, Sun H. TEP1, encoded by a candidate tumor suppressor locus, is a novel protein tyrosine phosphatase regulated by transforming growth factor beta. Cancer Res 1997; 57: 2124-2129.
2. Li J, Yen C, Liaw D, Podsypanina K, Bose S, Wang SI et al. PTEN, a putative protein tyrosine phosphatase gene mutated in human brain, breast, and prostate cancer. Science 1997; 275: 1943-1947.

3. Steck PA, Pershouse MA, Jasser SA, Yung WK, Lin H, Ligon AH et al. Identification of a candidate tumour suppressor gene, MMAC1, at chromosome 10q23.3 that is mutated in multiple advanced cancers. Nat Genet 1997; 15: 356-362.

4. Cantley LC, Neel BG. New insights into tumor suppression: PTEN suppresses tumor formation by restraining the phosphoinositide 3-kinase/AKT pathway. Proc Natl Acad Sci USA 1999; 96: 4240-4245.

5. Di Cristofano A, Pandolfi PP. The multiple roles of PTEN in tumor suppression. Cell 2000; 100: 387-390.

6. Maehama T, Taylor GS, Dixon JE. PTEN and myotubularin: novel phosphoinositide phosphatases. Annu Rev Biochem 2001; 70: 247-279.

7. Wishart MJ, Dixon JE. PTEN and myotubularin phosphatases: from 3-phosphoinositide dephosphorylation to disease. Trends Cell Biol 2002; 12: 579-585.

8. Bellacosa A, Testa JR, Staal SP, Tsichlis PN. A retroviral oncogene, akt, encoding a serine-threonine kinase containing an SH2-like region. Science 1991; 254: 274-277.

9. Chang HW, Aoki M, Fruman D, Auger KR, Bellacosa A, Tsichlis PN et al. Transformation of chicken cells by the gene encoding the catalytic subunit of PI 3-kinase. Science 1997; 276: $1848-1850$.

10. Jimenez C, Jones DR, Rodriguez-Viciana P, Gonzalez-Garcia A, Leonardo E, Wennstrom S et al. Identification and characterization of a new oncogene derived from the regulatory subunit of phosphoinositide 3-kinase. EMBO J 1998; 17: 743-753.

11. Staal SP. Molecular cloning of the akt oncogene and its human homologues AKT1 and AKT2: amplification of AKT1 in a primary human gastric adenocarcinoma. Proc Natl Acad Sci USA 1987; 84: 5034-5037.

12. Hlobilkova A, Guldberg P, Thullberg M, Zeuthen J, Lukas J, Bartek J. Cell cycle arrest by the PTEN tumor suppressor is target cell specific and may require protein phosphatase activity. Exp Cell Res 2000; 256: 571-577.

13. Moussavi M, Fazli L, Tearle H, Guo Y, Cox M, Bell J et al. Oncolysis of prostate cancers induced by vesicular stomatitis virus in PTEN knockout mice. Cancer Res 2010; 70: 1367-1376.

14. Raftopoulou M, Etienne-Manneville S, Self A, Nicholls S, Hall A. Regulation of cell migration by the C2 domain of the tumor suppressor PTEN. Science 2004; 303: 1179-1181.

15. Shelly S, Lukinova N, Bambina S, Berman A, Cherry S. Autophagy is an essential component of Drosophila immunity against vesicular stomatitis virus. Immunity 2009; 30: 588-598.

16. Shen WH, Balajee AS, Wang J, Wu H, Eng C, Pandolfi PP et al. Essential role for nuclear PTEN in maintaining chromosomal integrity. Cell 2007; 128: 157-170.

17. Tamura M, Gu J, Matsumoto K, Aota S, Parsons R, Yamada KM. Inhibition of cell migration, spreading, and focal adhesions by tumor suppressor PTEN. Science 1998; 280: 1614-1617.

18. Weng LP, Gimm O, Kum JB, Smith WM, Zhou XP, Wynford-Thomas D et al. Transient ectopic expression of PTEN in thyroid cancer cell lines induces cell cycle arrest and cell type-dependent cell death. Hum Mol Genet 2001; 10: 251-258.

19. Tamguney T, Stokoe D. New insights into PTEN. J Cell Sci 2007; 120: 4071-4079.

20. Hay RT. SUMO: a history of modification. Mol Cell 2005; 18: 1-12.

21. Meulmeester $\mathrm{E}$, Kunze M, Hsiao HH, Urlaub H, Melchior F. Mechanism and consequences for paralog-specific sumoylation of ubiquitin-specific protease 25. Mol Cell 2008; 30: 610-619.

22. Bergink S, Jentsch S. Principles of ubiquitin and SUMO modifications in DNA repair. Nature 2009; 458: 461-467.

23. Schimmel J, Larsen KM, Matic I, van Hagen M, Cox J, Mann M et al. The ubiquitinproteasome system is a key component of the SUMO-2/3 cycle. Mol Cell Proteomics 2008; 7: 2107-2122.

24. Ulrich HD. Mutual interactions between the SUMO and ubiquitin systems: a plea of no contest. Trends Cell Biol 2005; 15: 525-532.

25. Desterro JM, Rodriguez MS, Hay RT. SUMO-1 modification of IkappaBalpha inhibits NF-kappaB activation. Mol Cell 1998; 2: 233-239.

26. Lin X, Liang M, Liang YY, Brunicardi FC, Feng XH. SUMO-1/Ubc9 promotes nuclear accumulation and metabolic stability of tumor suppressor Smad4. J Biol Chem 2003; 278: 31043-31048.

27. Huang TT, Wuerzberger-Davis SM, Wu ZH, Miyamoto S. Sequential modification of NEMO/IKKgamma by SUMO-1 and ubiquitin mediates NF-kappaB activation by genotoxic stress. Cell 2003; 115: 565-576.

28. Lallemand-Breitenbach V, Jeanne M, Benhenda S, Nasr R, Lei M, Peres L et al. Arsenic degrades PML or PML-RARalpha through a SUMO-triggered RNF4/ubiquitin-mediated pathway. Nat Cell Biol 2008; 10: 547-555.

29. Tatham MH, Geoffroy MC, Shen L, Plechanovova A, Hattersley N, Jaffray EG et al. RNF4 is a poly-SUMO-specific E3 ubiquitin ligase required for arsenic-induced PML degradation. Nat Cell Biol 2008; 10: 538-546.

30. Weisshaar SR, Keusekotten K, Krause A, Horst C, Springer HM, Gottsche K et al. Arsenic trioxide stimulates SUMO-2/3 modification leading to RNF4-dependent proteolytic targeting of PML. FEBS Lett 2008; 582: 3174-3178.

31. Waite KA, Eng C. From developmental disorder to heritable cancer: it's all in the BMP/TGFbeta family. Nat Rev Genet 2003; 4: 763-773. 
32. Trotman LC, Wang X, Alimonti A, Chen Z, Teruya-Feldstein J, Yang $\mathrm{H}$ et al. Ubiquitination regulates PTEN nuclear import and tumor suppression. Cell 2007; 128: 141-156.

33. Ginn-Pease ME, Eng C. Increased nuclear phosphatase and tensin homologue deleted on chromosome 10 is associated with G0-G1 in MCF-7 cells. Cancer Res 2003; 63: 282-286.

34. Wimmer P, Schreiner S, Dobner T. Human pathogens and the host cell SUMOylation system. J Virol 2012; 86: 642-654.

35. Chelbi-Alix MK, Quignon F, Pelicano L, Koken MH, de The $\mathrm{H}$. Resistance to virus infection conferred by the interferon-induced promyelocytic leukemia protein. J Virol 1998; 72 1043-1051

36. Song MS, Salmena L, Carracedo A, Egia A, Lo-Coco F, Teruya-Feldstein J et al. The deubiquitinylation and localization of PTEN are regulated by a HAUSP-PML network. Nature 2008; 455: 813-817.

37. Boggio R, Colombo R, Hay RT, Draetta GF, Chiocca S. A mechanism for inhibiting the SUMO pathway. Mol Cell 2004; 16: 549-561.

38. Colombo R, Boggio R, Seiser C, Draetta GF, Chiocca S. The adenovirus protein Gam interferes with sumoylation of histone deacetylase 1. EMBO Rep 2002; 3: 1062-1068

39. Everett RD, Freemont $P$, Saitoh $H$, Dasso M, Orr A, Kathoria $M$ et al. The disruption of ND10 during herpes simplex virus infection correlates with the Vmw110- and proteasomedependent loss of several PML isoforms. J Virol 1998; 72: 6581-6591.

40. Muller S, Dejean A. Viral immediate-early proteins abrogate the modification by SUMO-1 of PML and Sp100 proteins, correlating with nuclear body disruption. J Virol 1999; 73: 5137-5143.

41. Ahn JH, Brignole EJ 3rd, Hayward GS. Disruption of PML subnuclear domains by the acidic IE1 protein of human cytomegalovirus is mediated through interaction with PML and may modulate a RING finger-dependent cryptic transactivator function of PML. Mol Cell Bio 1998: 18: 4899-4913.

42. Chang PC, Izumiya Y, Wu CY, Fitzgerald LD, Campbell M, Ellison TJ et al. Kaposi's sarcoma-associated herpesvirus KSHV) encodes a SUMO E3 ligase that is SIMdependent and SUMO-2/3-specific. J Biol Chem 2010; 285: 5266-5273.

43. Hwang J, Kalejta RF. In vivo analysis of protein sumoylation induced by a viral protein: Detection of HCMV pp71-induced Daxx sumoylation. Methods 2011; 55: 160-165.

44. Kubota T, Matsuoka M, Chang TH, Tailor P, Sasaki T, Tashiro M et al. Virus infection triggers SUMOylation of IRF3 and IRF7, leading to the negative regulation of type interferon gene expression. J Biol Chem 2008; 283: 25660-25670.

45. Pennella MA, Liu Y, Woo JL, Kim CA, Berk AJ. Adenovirus E1B 55-kilodalton protein is a p53-SUM01 E3 ligase that represses p53 and stimulates its nuclear export through interactions with promyelocytic leukemia nuclear bodies. J Virol 2010; 84: 12210-12225.

46. Rahdar M, Inoue T, Meyer T, Zhang J, Vazquez F, Devreotes PN. A phosphorylationdependent intramolecular interaction regulates the membrane association and activity of the tumor suppressor PTEN. Proc Natl Acad Sci USA 2009; 106: 480-485.

47. Geiss-Friedlander R, Melchior F. Concepts in sumoylation: a decade on. Nat Rev Mol Cell Biol 2007; 8: 947-956.
48. Kerscher $\mathrm{O}$, Felberbaum R, Hochstrasser M. Modification of proteins by ubiquitin and ubiquitin-like proteins. Annu Rev Cell Dev Biol 2006; 22: 159-180.

49. Verger A, Perdomo J, Crossley M. Modification with SUMO. A role in transcriptional regulation. EMBO Rep 2003; 4: 137-142.

50. Chugh P, Bradel-Tretheway B, Monteiro-Filho CM, Planelles V, Maggirwar SB, Dewhurst S et al. Akt inhibitors as an HIV-1 infected macrophage-specific anti-viral therapy. Retrovirology 2008; 5 : 11.

51. Chung TW, Lee YC, Ko JH, Kim CH. Hepatitis B Virus X protein modulates the expression of PTEN by inhibiting the function of p53, a transcriptional activator in liver cells. Cancer Res 2003; 63: 3453-3458.

52. Grandvaux N, tenOever BR, Servant MJ, Hiscott J. The interferon antiviral response: from viral invasion to evasion. Curr Opin Infect Dis 2002; 15: 259-267.

53. Muller U, Steinhoff U, Reis LF, Hemmi S, Pavlovic J, Zinkernagel RM et al. Functional role of type I and type II interferons in antiviral defense. Science 1994; 264: 1918-1921.

54. Sen GC. Viruses and interferons. Annu Rev Microbiol 2001; 55: 255-281.

55. Huang J, Yan J, Zhang J, Zhu S, Wang Y, Shi T et al. SUMO1 modification of PTEN regulates tumorigenesis by controlling its association with the plasma membrane. Nat Commun 2012; 3: 911.

56. Di Cristofano A, Pesce B, Cordon-Cardo C, Pandolfi PP. Pten is essential for embryonic development and tumour suppression. Nat Genet 1998; 19: 348-355.

57. Ramaswamy S, Nakamura N, Vazquez F, Batt DB, Perera S, Roberts TM et al. Regulation of G1 progression by the PTEN tumor suppressor protein is linked to inhibition of the phosphatidylinositol 3-kinase/Akt pathway. Proc Natl Acad Sci USA 1999; 96 : 2110-2115

58. Vertegaal AC, Andersen JS, Ogg SC, Hay RT, Mann M, Lamond Al. Distinct and overlapping sets of SUMO-1 and SUMO-2 target proteins revealed by quantitative proteomics. Mol Cell Proteomics 2006; 5: 2298-2310.

59. Campagna M, Herranz D, Garcia MA, Marcos-Villar L, Gonzalez-Santamaria J, Gallego P et al. SIRT1 stabilizes PML promoting its sumoylation. Cell Death Differ 2011; 18: 72-79.

60. Marcos-Villar L, Lopitz-Otsoa F, Gallego P, Munoz-Fontela C, Gonzalez-Santamaria J Campagna $\mathrm{M}$ et al. Kaposi's sarcoma-associated herpesvirus protein LANA2 disrupts PML oncogenic domains and inhibits PML-mediated transcriptional repression of the survivin gene. J Virol 2009; 83: 8849-8858.

Cell Death and Disease is an open-access journal published by Nature Publishing Group. This work is licensed under the Creative Commons Attribution-NonCommercial-No Derivative Works 3.0 Unported License. To view a copy of this license, visit http://creativecommons.org/licenses/by-nc-nd/3.0/

Supplementary Information accompanies the paper on Cell Death and Disease website (http://www.nature.com/cddis) 